\title{
A Survey on Spectrum Mobility in Cognitive Radio Network
}

\author{
Sudhani Verma \\ M. Tech Research Scholar, \\ Department of CSE \\ MANIT Bhopal, \\ Madhya Pradesh
}

\author{
Meenu Chawla, \\ Professor, \\ Department of CSE \\ MANIT Bhopal, \\ Madhya Pradesh
}

\begin{abstract}
Cognitive radio technology is an emerging technology which would enable a set of secondary users (SU) to opportunistically use the spectrum allocated to a primary user (PU) and has potential to serve as a solution to spectrum inefficiency and spectrum shortage problems. However, SUs face number of challenges based on the fluctuating nature of the available spectrum. When PU arrives on a specific frequency band, any SU occupying this band should free the channel for PUs which is referred as Spectrum Mobility. It is an important but unexplored event in cognitive radio network. This paper is a brief overview on the reason, mechanism, challenges and their solutions in spectrum mobility.
\end{abstract}

Keywords: Cognitive Radio Network (CRN), Spectrum Mobility/Handoff.

\section{INTRODUCTION}

The rapid growth in wireless technologies has led to a huge increase in spectrum demand. On the contrary as per a report given by Federal Communications Commission (FCC) [1], allocated spectrum is under-utilized due to the inefficient fixed spectrum allocation policy. This limited available spectrum and the inefficiency in the spectrum usage necessitates a new communication paradigm to exploit the existing wireless spectrum opportunistically by access to the licensed bands without interfering with the licensed users. The licensed user referred as primary user and the unlicensed user are referred as secondary user and this new networking paradigm is referred as Dynamic Spectrum Access (DSA) or cognitive radio networks (CRN) [2] (These terms can be used interchangeably). Cognitive radio is a new approach to reduce the spectrum scarcity by allowing the secondary users to temporarily utilize the licensed spectrum unoccupied by the primary user.

The cognitive radio technology will enable the users to determine which portions of the spectrum is available and detect the presence of licensed users when a user operates in a licensed band known as spectrum sensing. Select the best available channel through spectrum management. Coordination of the access to this channel with other users known as spectrum sharing, and if band is found to be occupied by a licensed user, the CR user moves to another spectrum hole to avoid interference known as spectrum mobility. Through cognitive capability and reconfigurability prime objective of cognitive radio network to obtain best available channel is achieved which is discussed in [3] also architecture and proper functioning of CR transceiver is explained. Due to the broad range of available spectrum, distinct Quality-of-Service (QoS) requirements, number of research challenges exist which are discussed in [4]. The mobile terminals roam between wireless architectures along the available spectrum pool, this introduces more challenges. Most of the spectrum is already assigned to licensed/primary user. The important challenge is to share the licensed spectrum without interfering with the transmission of the licensed users. The cognitive radio facilitates the unlicensed user to use temporally unused spectrum, referred as spectrum hole or white space. Since the licensed/ primary user has higher priority than secondary user, the unlicensed/secondary user is required to give up the occupied channel when the primary user appears and determine a new suitable channel to resume its unfinished transmission. This action is named as spectrum handoff or mobility and it is less explored in the research community in comparison with other major functionalities namely spectrum sensing, sharing and decision.

\section{SPECTRUM MOBILITY IN CRN}

The concept of spectrum handoff/mobility in CR networks is different from the classical handoff mechanisms in wireless networks. Based on priority two different types of users are studied in Spectrum handoff/mobility. High priority user (also called as Primary Users) has the right to interrupt the transmission of the low-priority users (also called as Secondary Users) and ask them to leave the channel even though lowpriority user has respectable signal strength. All users in classical handoff have the same priorities and the decision of changing channels is made mainly due to the deterioration of the current channel signal quality.

\section{PROCESS OF SPECTRUM MOBILITY/HANDOFF}

In CR systems, the sudden appearance of a PU on a band occupied by a SU triggers the cognitive user to leave this band as soon as possible. The SU would then try to regain the medium through one of the following three actions: (i) until the PU finishes its transmission SU will stay in the original channel and postpones its transmission, (ii) select a channel from a list of previously sensed channels (predetermined spectrum handoff) or (iii) switching to a certain channel after immediate sensing (sensing-based spectrum handoff) and if SU fails in regaining the spectrum it is compelled to terminate its session. In fig 1(a) secondary users SU1 and SU2 communicate on channel Ch1. When primary user appears on Ch1 SUs pause its current communication as in fig 1(b). Now SU1 has two options i) SU1 and SU2 can resume its transmission on the selected target channel as in fig 1(c) or ii) it can also remain on the same channel and resume the transmission after the PU activity is over as in fig 1(d) this case is preferred if PU activity is small as it will reduce the number of handoff. A frame may 
be interrupted many times during its transmission hence spectrum handoff procedure may be executed many times.

SU1 wants to tranmit to SU2

Ch1 SU1 ==> SU2

Ch2

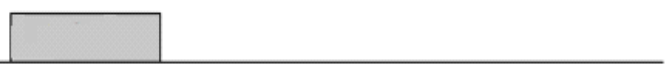

Fig 1(a): Transmission between SUs

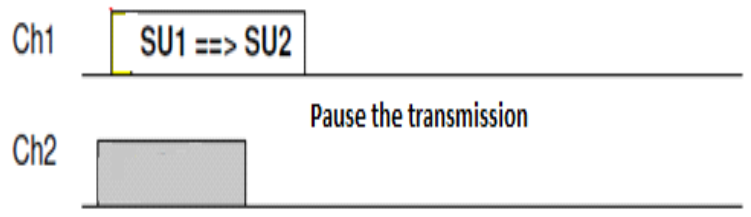

Fig 1(b): PU appears

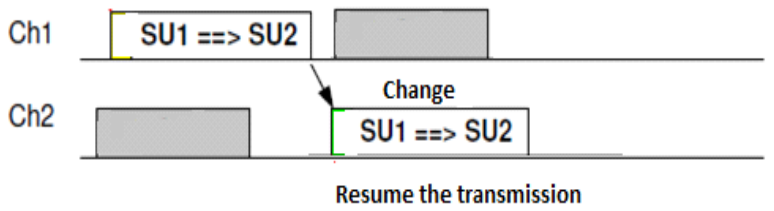

Fig 1(c): Resume transmission on selected channel

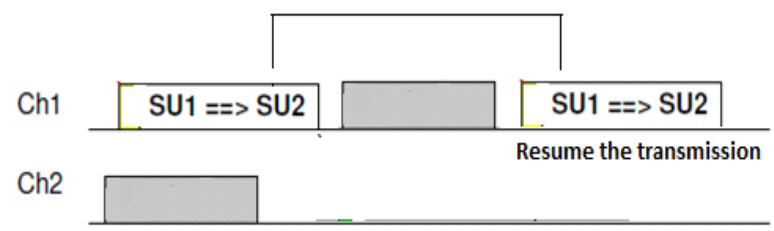

Fig 1(d): Resume transmission on the same channel

\section{IMPLEMENTATION STRATEGY OF SPECTRUM HANDOFF}

Design, analysis and optimization of DSA require versatile awareness. This includes knowledge of wireless communication and networking, signal processing techniques (to estimate parameters), filtering and prediction (to gain knowledge of radio environment), machine learning techniques (to learn, plan and optimize the decision of DSA), optimization techniques (to obtain the optimal dynamic spectrum management scheme). It also includes intelligent techniques based on fuzzy logic and decision theory.

In the process of spectrum handoff decisions are made based on incomplete and vague information available at the SU as a result of the spectrum sensing. An approach based on fuzzy logic has potential to make effective spectrum handoff decisions in a context which is characterized by incomplete, uncertain and heterogeneous information. In [5] an approach using Fuzzy Logic System (FLS) to control the spectrum access is proposed which detects the effective spectrum access for secondary users via cognitive radio. In [6], [7] a decentralized architecture was considered, as solution to problem of the spectrum handoff decision, channel selection and channel estimation on the way how a SU makes this decision based on the information available from the spectrum sensing. To trigger spectrum handoff a simple and less complex fuzzy-based approach is suggested, which guarantees quick decisions so that SUs can autonomously and automatically make the decision to change its frequency channel. Fuzzy Logic is a simple way to arrive at a definite conclusion based upon ambiguous and missing input information but it is expensive as it requires extensive testing and system that use fuzzy logic do not have ability to learn and adapt after solving a problem as some expert system can.

Spectrum handoff helps secondary users to find appropriate target channels to resume the unfinished transmission. In general, according to the target channel selection methods, spectrum handoff mechanisms can be classified into: (i) proactive-sensing spectrum handoff; and (ii) reactive-sensing spectrum handoff. In proactive-sensing spectrum handoff, before transmission, secondary users select the target channels for spectrum handoff. Periodically secondary users observe all channels and channel usage statistics are obtained to find the candidate set of target channels for spectrum handoff. In reactive-sensing spectrum handoff, the target channels are searched by the on-demand strategy. In this case, the result from wideband sensing will be used to determine the target channel selection for spectrum handoff. In comparison to reactive-decision spectrum handoff, the proactive-decision spectrum handoff may be able to reduce handoff delay because the wideband sensing is not required here but the target channel which is pre-determined may be unavailable. The advantage of the reactive spectrum handoff is the accuracy of the selected target channel, but requires extra sensing time.

In CRN channel is allocated on the basis of priority to primary and secondary user, for modeling and analysis preemptive resume priority (PRP) M/G/1 queue model can be used. In [8] a comparison of reactive and proactive is done with the help of proposed PRP M/G/1 queue network model. A relationship between model and spectrum handoff is established and the transmission time for a frame in single and multiple handoffs is calculated, this transmission time is further used to calculate the latency in transmission for reactive and proactive mechanism. The same author has provided a modeling and analysis of proactive and reactive spectrum handoff separately in [9] and [10] respectively. For proactive in [9] a preemptive resume priority (PRP) $\mathrm{M} / \mathrm{G} / 1$ queuing network model is proposed. Based on this model, the total service time for various target channels sequences can be evaluated, and we can find the optimal target channels sequences. As mentioned above proactive handoff is having limitation of predetermined channel unavailability. A suboptimal greedy target channel selection scheme is proposed to reduce the complexity for finding optimal target channels. While in reactive decision spectrum handoff, handoff delay may be shorter because it can accurately find an idle channel through spectrum sensing but requires extra sensing time. In [10] the secondary user can reactively decide the target channel for spectrum handoff to resume its unfinished transmission. A Markov transition model is integrated with the preemptive resume priority (PRP) M/G/1 queuing network to characterize the effects of the sensing time, the channel switching time, the handshaking time on the handoff delay in CR networks is proposed.

In comparison to reactive, proactive has advantage of avoiding collision between the primary users and secondary users, it increases the throughput of the primary and secondary users, but requires complex algorithm to implement. In [11] a proactive spectrum handoff protocol based on the Greedy Channel Selection (GCS) which avoids collision between secondary users, also between primary and secondary users is proposed. Furthermore, the channel selection is distributed which results in higher throughput, and lower average service time. Random appearance of licensed users may lead to low 
throughput in communication of both licensed and unlicensed users. In [12] a proactive spectrum handoff framework for CR ad hoc networks is proposed. Channel switching policies and a proactive spectrum handoff protocol are suggested so that unlicensed users vacate a channel before a licensed user appears, to avoid unwanted interference. SUs equipped with the prediction capability can proactively predict the idleness probability of the spectrum band in the near future. Hence harmful interference between SUs and PUs can be avoided and SU throughput is increased.

Spectrum handoff procedures aim to help the secondary users to vacate the occupied licensed spectrum and find suitable target channel to resume the unfinished transmission and in proactive the decision is made on past usage of channel, future prediction of channel usage and on probability of idleness of channel. However there exists the instability due to prediction and probabilistic approach here.

\section{CHALLENGES AND SOLUTIONS IN SPECTRUM HANDOFF}

Spectrum handoff is indispensible event of transmission in cognitive environment and it should be handled properly. Reduce the handoff latency, find a proper set of channels to complete the unfinished transmission and to maintain the required quality of service are important challenges in spectrum mobility, discussed in [13] also there are several others challenges which are discussed in this section.

If presence of primary users is detected through collaborative effort of secondary users, this will hit on the power consumption of secondary users. Managing power is a critical factor in CR network and to identify the exact transition time when a secondary user needs to hand over the spectrum to the primary user is difficult. The broad range of available spectrum, undulating nature of the available spectrum, heterogeneous mobility events makes it more difficult to support seamless communications. Evaluating the suitability of a new available spectrum for usage and selecting the best channel among available multiple channels could also be very tough job to do. The channel's parameters in terms of channel width, bandwidth, rate, etc. need to be evaluated before taking a decision and this could be a complex process. In [14] a cellular architecture is assumed for CR and a spectrum aware mobility management scheme is proposed. Network architecture is introduced based on the spectrum pooling to mitigate heterogeneous spectrum availability. Another challenge is transport layer protocols' performance of a SU which could be degraded significantly as on PU arrival it tries to give up channel. The primary reason for most of packet losses is route failure due to mobility but TCP assumes that packet loss occurs because of congestion, so TCP will invoke congestion control mechanisms for packet loss which is actually caused by route failures, resulting in low throughput. In [15] a solution to the above problem is given by identifying the problems which make TCP implementation in CR different from that of conventional wireless network. A TCP rate adapting algorithm which ensures seamless spectrum handoff as PU appears is proposed.

New mobility and connection management approaches need to be designed to reduce delay and loss of information during spectrum handoff. Novel algorithms are required to ensure that applications do not suffer from severe performance degradation when they have to be transferred to another available frequency band due to the appearance of a primary user. This performance degradation could be due to link destruction and to preserve the quality of transmission, link maintenance is important. In [16] we can see the link maintenance probability can be achieved better if radio sensing time, erroneous channel selection probability and the number of handoff are reduced. If SU didn't get the channel for transmission it leads to forced termination of the session. To avoid this problem of forced termination in [17] virtual reservation is introduced as a new link maintenance strategy which reduces the forced termination probability of SU. It is a novel link maintenance strategy that aims to maximize the throughput of the cognitive network by utilizing the spectrum at most. Multiple handoffs for a user may reduce the quality of service for an interrupted user. An interrupted user is referred to that $\mathrm{SU}$ which has been interrupted by PU user, when it is utilizing a licensed band, and due to appearance of PU it has to pause its transmission and has to change their operating channel. To maintain a required level of quality of service (QoS) for SUs, an effective spectrum handoff procedure should be initiated. In [18] a priority based model is proposed where priority is given to interrupted user over new uninterrupted user. In this model the interrupted user are required to maintain a queue and wait until all other primary and secondary users receive their services. This wait will add extra time in their delay and hence increase handoff delay and service time. By maintaining a queue we can easily identify the interrupted user, so we can give them priority over uninterrupted user. This will reduce the handoff delay and maintain the required level of quality of service (QoS). To model the proposed work a preemptive resume priority (PRP) $\mathrm{M} / \mathrm{G} / 1$ queue model is used.

Spectrum handoff is time consuming process as SUs need to search for the available spectrum and reconfigure their operating frequency at the RF front-ends dynamically and abruptly whenever spectrum availability changes, due to which a significant switching latency also called spectrum handoff delay is introduced. It includes time taken in spectrum sensing, analysis, decision and spectrum switching. Hence it is better to reduce unnecessary spectrum handoffs. In [19] the SU decides to wait silently in their current channel until the PU departs, instead of immediately switching to another idle channel. This option would be favorable to the SU if the returned PU stays at the channel only for a short period of time. An expression for maximal waiting time for which the SUs should wait is derived in two cases when PU distribution is known and when it is unknown. Spectrum handoff may cause service interruptions or significant delays leading to the degradation of the quality of transmission. In [20] a novel technique that aims at reducing spectrum handoff delays while keeping the quality of the transmission acceptable is suggested. The significant step in attaining a good level of SU's service is to know how to select best channels. In the beginning the expected delay of application packets is computed as a function of the queue associated with a channel. The calculated delay reflects the status of the channel and its usability for transmitting packets, then this delay is used to calculate delay violation ratio that guides the spectrum handoff and selection decisions.

To reduce the handoff latency a list of backup channel is maintained so that SU can switch to one of the channels among the list quickly whenever handoff took place. In the first standard for CR networks [21] IEEE 802.22, a backup channel list is maintained to provide the maximum probability of finding an available spectrum band within the shortest time. In [19] and [20] the backup channels list attempt to minimize the switching delay which can be reduced if there are more idle channels in the backup channels list. A study on the utility of using additional backup channels at the time of handoff to 
alleviate the problem of fast successive handoffs is done but maintaining a backup channel list is itself a problem.

\section{CONCLUSION}

Cognitive radio (CR) has the potential for resolving the spectrum scarcity issue in wireless communications. Spectrum handoff is very important but is the least explored area in Cognitive radio network. We have tried to mention the mechanism and reason of spectrum handoff and also some important challenges and their solutions. The research which has been done and explained in spectrum handoff area is based on prediction and probabilistic approach which in a way is unavoidable because of uncertain behavior of Primary users. The prime objective of handoff is to provide smooth and fast transmission to secondary users. New mobility and connection management approaches need to be designed to reduce delay and loss of information during spectrum handoff. A mechanism has to be there to reduce spectrum handoff latency, a list of backup channel is maintained for this purpose but maintaining and updating that is itself a challenge. Future work includes to design a framework for spectrum handoff which is less probability and prediction based and to understand the mechanism of backup channel list and how we can utilize this list at most to provide a smooth and fast transmission.

\section{REFRENCES}

[1] FCC, ET Docket No 03-222 Notice of proposed rulemaking and order, December 2003.

[2] Akyildiz, Ian F., Won-Yeol Lee, and Kaushik R. Chowdhury. "CRAHNs: Cognitive radio ad hoc networks." Ad Hoc Networks 7.5 (2009): 810-836.

[3] Akyildiz, Ian F., et al. "NeXt generation/dynamic spectrum access/cognitive radio wireless networks: a survey." Computer Networks 50.13 (2006): 2127-2159.

[4] Van Tam Nguyen, Frederic Villain and Yann Le Guillou. "Cognitive Radio RF: Overview and Challenges."Hindwai Publishing Corporation VLSI design Volume 2012, Article ID716476, 13 pages.

[5] Kaniezhil. R, Daniel Nesa Kumar. C and Prakash. A, "Fuzzy Logic System for Opportunistic Spectrum Access using Cognitive Radio", International Journal of Scientific \& Engineering Research Vol. 10, Issue 1, No 1, January 2013

[6] Giupponi, Lorenza, and Ana I. Pérez-Neira. "Fuzzy-based spectrum handoff in cognitive radio networks." Cognitive Radio Oriented Wireless Networks and Communications, 2008. CrownCom 2008. 3rd International Conference on. IEEE, 2008.

[7] R. Kaniezhil, Dr. C. Chandrasekar, "Comparing Spectrum Utilization using Fuzzy Logic System for Heterogeneous Wireless Networks via Cognitive Radio", International Journal of Scientific \& Engineering Research, Volume 3, Issue 7,July 2012, pp.1 - 10.

[8] Wang, Li-Chun, and Chung-Wei Wang. "Spectrum handoff for cognitive radio networks: Reactive-sensing or proactive-sensins?" Performance, Computing and Communications Conference, 2008. IPCCC 2008. IEEE International. IEEE, 2008.
[9] C-W. Wang and L-C. Wang, "Modeling and Analysis for Proactive-decision Spectrum Handoff in Cognitive Radio Networks", IEEE International Conference on Communications, June 2009

[10] Wang, Chung-Wei, Li-Chun Wang, and Fumiyuki Adachi. "Modeling and analysis for reactive-decision spectrum handoff in cognitive radio networks."Global Telecommunications Conference (GLOBECOM 2010), 2010 IEEE. IEEE, 2010.

[11] Morteza Mehrnoush, V. T. Vakili. "proactive srv spectrum handoff protocol based on gcs scheme in cognitive radio adhoc network." International Journal of Power Control Signal and Computation (IJPCSC) Vol. 5. No.1. pp.1-08, Jan-March 2013

[12] Song, Yi, and Jiang Xie. "On the Spectrum Handoff for Cognitive Radio Ad Hoc Networks without Common Control Channel." Cognitive Radio Mobile Ad Hoc Networks. Springer New York, 2011. 37-74.

[13] Salem, Tarek M., et al. "Efficient Spectrum Management: Challenges and Solution." http://www.academia.edu/7684823/Efficient_S pectrum_Management_Challenges_and_Solutions

[14] Lee, Won-Yeol, and Ian F. Akyildiz. "Spectrum-aware mobility management in cognitive radio cellular networks." Mobile Computing, IEEE Transactions on 11.4 (2012): 529-542.

[15] Balogun, Victor. "Challenges of Spectrum handoff in Cognitive radio networks."Pacific Journal of Science and Technology 11 (2010): 304-314.

[16] Wang, Li-Chun, and Chen Anderson. "On the performance of spectrum handoff for link maintenance in cognitive radio." Wireless Pervasive Computing, 2008. ISWPC 2008. 3rd International Symposium on. IEEE, 2008.

[17] Abdel-Hamid, Ayman T., Ahmed H. Zahran, and Tamer ElBatt. "Improved spectrum mobility using virtual reservation in collaborative cognitive radio networks." Computers and Communications (ISCC), 2013 IEEE Symposium on. IEEE, 2013.

[18] Zahed, Salah, Irfan Awan, and Andrea Cullen. "Analytical modeling for spectrum handoff decision in cognitive radio networks." Simulation Modelling Practice and Theory 38 (2013): 98-114.

[19] Zhang, Caoxie, and Kang G. Shin. "What Should Secondary Users Do Upon Incumbents' Return?" Selected Areas in Communications, IEEE Journal on31.3 (2013): 417-428.

[20] Lertsinsrubtavee, Adisorn, Naceur Malouch, and Serge Fdida. "Controlling spectrum handoff with a delay requirement in cognitive radio networks."Computer Communications and Networks (ICCCN), 201221 st International Conference on. IEEE, 2012.

[21] Cordeiro, Carlos, et al. "IEEE 802.22: the first worldwide wireless standard based on cognitive radios." New Frontiers in Dynamic Spectrum Access Networks, 2005. DySPAN 2005. 2005 First IEEE International Symposium on. IEEE, 2005. 